\title{
APPROXIMATION OF IRRATIONALS
}

\author{
MALVINA BAICA \\ University of Wisconsin-Whitewater \\ Department of Mathematics \\ and Computer Science \\ Whitewater, Wisconsin 53190 U.S.A. \\ (Received January 13, 1984 and in revised form February 10, 1985)
}

ABSTRACT. The author uses a new modification of Jacobi-Perron Algorithm which holds for complex fields of any degree (abbr. ACF), and defines it as Generalized Euclidean Algorithm (abbr. GEA) to approximate irrationals.

This paper deals with approximation of irrationals of degree $n=2,3,5$. Though approximations of these irrationals in a variety of patterns are known, the results are new and practical, since there is used an algorithmic method.

KEY WORDS AND PHRASES. Irrationals, Jacoli-Perron Algorithm labbr. JPA), An Algorithm in a Complex Field (abbr. ACF), Generalized

Euclidean Algorithm (abbr. GEA).

1980 MATHEMATICS SUBJECT CLASSIFICATION CODE. 10A30, $10 F 05$.

0. INTRODICTION.

This paper deals with approximation of algebraic irrationals of degree $n=2,3,5$.

Most simple rational fractions are approximating irrationals, especially of the type $\sqrt{D^{2}+1}, \sqrt[3]{D^{3}}+1$, and $\sqrt[5]{D^{5}+1}$, with $D=1$, leading to the most simple form of $\sqrt{2}, \sqrt[3]{2}$ and $\sqrt[5]{2}$. Though approximations of these irrationals in a variety of patterns are known, the results under discussion here are new and practical.

The main algebraic machine-tool which is the starting point and the main ingredient of this paper is an algorithm which, for algebraic real numbers, can be regarded as a generalization of Euclidean Algorithm (abbr. GEA).

1. THE GEA

The invention of the Euclidean Algorithm was one of the great achievements of ancient mathematics. Its special power rests with its periodicity which, regretfully, holds only in real quadratic fields. Jacobi [1] generalized it for the cubic real fields, and Perron [2] 
for $n$-th degree real algebraic fields. Since then, various modifications of the algorithm was given by prominent authors in the modern mathematical world. The author [3] used a modification which holds for complex fields of any degree, abbr. ACF. In the sequel, it will be called the "Generalized Euclidean Algorithm", abbr. GEA. It proceeds as follows:

DEFINITION 1 . Let

$$
a^{(0)}=\left(a_{1}^{(0)}, a_{2}^{(0)}, \ldots, a_{n-1}^{(0)}\right) \in E_{n-1}, n \geq 1
$$

be a fixed, given vector, and

$$
\left\langle\mathrm{a}^{(\mathrm{v})}\right\rangle, \mathrm{v}=0,1, \ldots, \mathrm{a}^{(\mathrm{v})} \in \mathrm{E}_{\mathrm{n}-1}
$$

a sequence of vectors in $E_{n-1}$ either given by some rule or calculated from $a^{(0)}$,

$$
a^{(v)}=\left(a_{1}^{(v)}, a_{2}^{(v)}, \ldots, a_{n-1}^{(v)}\right) \text {. }
$$

Let $\left\langle b^{(v)}\right\rangle, v=0,1, \ldots ; b^{(v)} \in E_{n-1}$ be another sequence of vectors in $E_{n-1}$, either given by some rule or calculated from $\langle a(v)\rangle$ with the formula

$$
\left.\begin{array}{rl}
a^{(v+1)}= & \left(a_{1}^{(v)}-b_{1}^{(v)}\right)^{-1}\left(a_{2}^{(v)}-b_{2}^{(v)}, \ldots, a_{n-1}^{(v)}-b_{n-1}^{(v)}, 1\right) \\
v & =0,1, \ldots
\end{array}\right\}
$$

we say that the GEA holds for $a^{(0)} \cdot b^{(v)}$ is called the "companion vectorn of $a^{(\mathrm{V})}$.

DEFINITION 2. We form the numbers

$$
\begin{aligned}
A_{i}^{(v)} & =\delta_{i}^{(v)}, \text { the Kronecker delta } \\
i, v & =0,1, \ldots, n-1
\end{aligned}
$$

Let a GEA of $a^{(0)}$ hold. The recursion formula

$$
A_{i}^{(n+v)}=\sum_{j=0}^{n-1} b_{j}^{(v)} A_{i}^{(v+j)}, b_{0}^{(v)}=1 ; \quad v=0,1, \ldots
$$

generates the "matricians" of the GEA of a ${ }^{(0)}$. L. Bernstein [4] has proved the following formulas for the real algebraic fields, and the author proved [3] that they are also true for the complex fields.

$$
\begin{aligned}
& \left|\begin{array}{l}
A_{0}^{(v)}, A_{0}^{(v+1)}, \ldots, A_{0}^{(v+n-1)} \\
A_{1}^{(v)}, A_{1}^{(v+1)}, \ldots, A_{1}^{(v+n-1)} \\
\hdashline A_{n-1}^{(v)}, A_{n-1}^{(v+1)}, \ldots, A_{n-1}^{(v+n-1)}
\end{array}\right|=(-1)^{v(n-1)} \\
& \left.a_{i}^{(0)}=\frac{\sum_{j=1}^{n-1} a_{j}^{(v)} A_{i}^{(v+j)}}{\sum_{j=0}^{n=1} a_{j}^{(v)} A_{O}^{(v+j)}} ; a_{0}^{(v)}=1, \begin{array}{l}
i=0,1, \ldots ; \\
v=0,1, \ldots
\end{array}\right\}
\end{aligned}
$$


DEFINITION 3. Let the GEA of an a ${ }^{(0)}$ hold. If there exist two numbers, $s \geq 0, t \geq 1 ; s, t \in N$ such that $a^{(s+t)}=a^{(t)}$, then the GEA of this a $(0)$ is called "periodic". If contemporarily $\min s=m \geq 0$, $\min t=\ell \geq 1$, then $\langle a(v)\rangle, v=0,1, \ldots, m-1,\langle a(v)\rangle, v=m, m+1, \ldots$, $m+\ell-1$ are called respectively the "primitive preperiod $n$ and the "primitive period" of the GEA of $a^{(0)}$. If $m=0$, the GEA of $a^{(0)}$ is called "purely periodic".

$\mathrm{m}$ and $\ell$ are called respectively the lengths of the primitive preperiod and primitive period.

Perron [5] proved the following theorem which is a generalization of the original theorem on convergence of simple continued fractions. In our terminology this would yield

THEOREM 1. Let a GEA of some $a^{(0)} \in E_{n-1}$ hold. If the components of all companion vectors are positive and the GEA of this a $(0)$ is periodic then

$$
a_{i}^{(0)}=\lim _{v \rightarrow \infty} \frac{A_{i}^{(v)}}{A_{0}^{(v)}}, i=0,1, \ldots
$$

where $A_{i}^{(v)},(i=0,1, \ldots, n-1 ; v=0,1, \ldots)$ are the matricians of the GEA of ${ }^{1}(0)$.

${ }_{a}^{(0)}$. The fractions $\frac{A_{i}^{(v)}}{A_{0}^{(v)}}$ are called the "convergents" of the GEA of

The reader should note that if the GEA of some a ${ }^{(0)}$ is periodic then there exists an $a^{(v)}$ in this GEA such that the GEA of $a^{(v)}$ is purely periodic. With this in mind the author [3] has proved

THEOREM 2. Let

$\mathrm{w}$ an $\mathrm{n}$-th degree integer $(\mathrm{n} \geq 2)$, and $\mathrm{a}^{(0)}$ a fixed vector such that

$$
\left.\begin{array}{l}
a^{(0)}=\left(a_{1}^{(0)}(w), a_{2}^{(0)}(w), \ldots, a_{n-1}^{(0)}(w)\right) \\
a_{i}^{(0)}(w) \text { algebraic integers }(i=1, \ldots, n-1) .
\end{array}\right\}
$$

Let the GEA of $a^{(0)}$ be purely periodic with length of the primitive period $=\ell$. Let the components of the companion vectors be algebraic integers. Then

$$
\left.\begin{array}{c}
\mathrm{A}_{0}^{(\mathrm{v} \ell)}+\mathrm{a}_{1}^{(0) \mathrm{A}_{\mathrm{O}}(\mathrm{v} \ell+1)}+\mathrm{a}_{2}^{(0)} \mathrm{A}_{\mathrm{O}}^{(\mathrm{v} \ell+2)}+\cdots+\mathrm{a}_{n-1}^{(0)} \mathrm{A}_{0}^{(v \ell+n-1)} \\
v=1,2, \ldots
\end{array}\right\}
$$

and units, namely the $v$-th powers of

$$
\mathrm{A}_{0}^{(l)}+\mathrm{a}_{1}^{(0)} \mathrm{A}_{0}^{(l+1)}+\mathrm{a}_{2}^{(0)} \mathrm{A}_{0}^{(l+2)}+\cdots+\mathrm{a}_{\mathrm{n}-1}^{(0)} \mathrm{A}_{0}^{(l+n-1)} .
$$

In this context we shall need the formula which was also used by the author in her paper [5], viz. 


$$
\prod_{i=1}^{k} a_{n-1}^{(i)}=A_{0}^{(k)}+a_{1}^{(k)} A_{0}^{(k+1)}+\cdots+a_{n-1}^{(k)} A_{0}^{(k+n-1)} \text {. }
$$

If the GEA of $a^{(0)}$ in $E_{n-1}$ is purely periodic with length of the primitive period $\ell$, then it follows from (1.11)

$$
e=\prod_{i=0}^{\ell-1} a_{n-1}^{(i)}=\sum_{j=0}^{n-1} a_{j}^{(0)} A_{0}^{(\ell+j)} \text {, e a unit }
$$

since in this case $a_{n-1}^{(\ell)}=a_{n-1}^{(0)}$.

We also have the formula, in virtue of (1.10)

$$
\left.\begin{array}{c}
e^{f}=A_{0}^{(f \ell)}+a_{1}^{(0)} A_{0}(f \ell+1)+\cdots+a_{n-1}^{(0)} A_{0}(f \ell+n-1) \\
f=1,2, \ldots
\end{array}\right\}
$$

\section{APPROXIMATION OF IRRATIONALS - CASE $n=2$}

Though this case is well known from the expansion of real quadratic irrationals as simple continued fractions, we shall include it in our discussion.

Let

$$
w=\sqrt{D^{2}+1}, D \in N \text {, w a quadratic irrational. }
$$

That $w$ is irrational (for $D>0$ ) is banal.

We choose the fixed vector

$$
\mathrm{a}^{(0)}=\mathrm{w}+\mathrm{D} \text {, }
$$

since here $n-1=1$. Thus $a_{1}^{(0)}=a_{n-1}^{(0)}$, and we shall generally denote

$$
a^{(v)}=a_{v}, v=0,1, \ldots ; a_{v}=a_{v}(w) \text { for all GEA of } a^{(0)} \text {. }
$$

In conformity with (2.3) we shall also denote

$$
b^{(v)}=b_{v}, \quad v=0,1, \ldots .
$$

For the calculation of the companion vectors we use the rule

$$
b^{(v)}=b_{v}=a_{v}(D), v=0,1, \ldots
$$

and have

$$
\mathrm{b}_{\mathrm{O}}=(\mathrm{w}+\mathrm{D})_{\mathrm{w}=\mathrm{D}}=2 \mathrm{D}
$$

hence, by (2.1)

$$
a_{1}=[(w+D)-2 D]^{-1} \cdot 1=(w-D)^{-1}=w+D
$$

since $(w-D)^{-1}=(w+D)$ from $w^{2}-D^{2}=1$. Thus

$$
a_{0}=a_{1}=\cdots=a_{v}, \quad v=0,1, \ldots
$$

and the GEA of $a_{O}=w+D$ is purely periodic with length of the primitive period $\ell=1$. Further, in this case,

$$
\left[a_{v}\right]=[w+D]=[w]+D=2 D=b_{v}
$$

the GEA of $w+D$ coincides with the Euclidean algorithm, and we have, in 
the notation of continued fractions

$$
a_{0}=w+D=[\overline{2 D}] \text {. }
$$

For the calculation of the matricians of $a^{(0)}$ we have from (1.3) and (1.4)

$$
\left.\begin{array}{rl}
A_{0}^{(0)} & =1, A_{0}^{(1)}=0, A_{0}^{(n+2)}=A_{0}^{(n)}+2 D A_{0}^{(n+1)}, \\
A_{1}^{(0)} & =0, A_{1}^{(1)}=1, A_{1}^{(n+2)}=A_{1}^{(n)}+2 D A_{1}^{(n+1)}, \\
n & =0,1, \ldots .
\end{array}\right\}
$$

Formula (1.6) yields

$$
\begin{aligned}
& w+D=\frac{A_{1}^{(v)}+(w+D) A_{1}^{(v+l)}}{A_{0}^{(v)}+(w+D) A_{0}^{(v+1)}} \\
& (w+D) A_{0}^{(v)}+(w+D)^{2} A_{0}^{(v+1)}=A_{1}^{(v)}+(w+D) A_{1}^{(v+l)},
\end{aligned}
$$

and comparing in (2.10) coefficients of $w$ (namely the highest irrational power of $w)$, we obtain

$$
A_{1}^{(v+l)}=A_{0}^{(v)}+2 D A_{0}^{(v+1)}=A_{1}^{(v+2)}
$$

\section{EXPLICIT REPRESENTATION OF THE MATRICIANS}

We shall give an explicit representation of $A_{0}^{(v)}(v=2,3, \ldots)$. By formula (2.12) which, because of (2.11) will also provide an explicit representation of $A_{l}^{(v)}(v=2,3, \ldots)$, we obtain from (2.8), by means of Euler's function

$$
\begin{aligned}
& \sum_{i=0}^{\infty} A_{0}^{(i)} x^{i}=A_{0}^{(0)}+A_{0}^{(1)} x+\sum_{i=2}^{\infty} A_{0}^{(i)} x^{i}= \\
& 1+\sum_{i=0}^{\infty} A_{0}^{(i+2)} x^{i+2}= \\
& =1+\sum_{i=0}^{\infty}\left(A_{0}^{(i)}+2 D A_{0}^{(i+1)}\right) x^{i+2}= \\
& =1+x^{2} \sum_{i=0}^{\infty} A_{0}^{(i)} x^{i}+2 D x \sum_{i=0}^{\infty} A_{0}^{(i+1)} x^{i+1}= \\
& =1+x^{2} \sum_{i=0}^{\infty} A_{0}^{(i)} x^{i}+2 D x\left(-A_{0}^{(0)}+\sum_{i=0}^{\infty} A_{0}^{(i)} x^{i}\right) .
\end{aligned}
$$

Hence

$$
\begin{aligned}
& \left(1-x^{2}-2 D x\right) \sum_{i=0}^{\infty} A_{O}^{(i)} x^{i}=1-2 D x, \\
& \sum_{i=0}^{\infty} A_{O}^{(i)} x^{i}=\frac{1-2 D x}{1-\left(x^{2}+2 D x\right)}=1+\frac{x^{2}}{1-\left(x^{2}+2 D x\right)},
\end{aligned}
$$




$$
\begin{aligned}
& A_{0}^{(0)}+A_{0}^{(I)} x+\sum_{i=2}^{\infty} A_{0}^{(i)} x^{i}=1+\sum_{i=0}^{\infty} A_{0}^{(i+2)} x^{i+2} \\
& =\frac{x^{2}}{1-\left(x^{2}+2 D x\right)}, \\
& \sum A_{0}^{(i+2)} x^{i}=\sum_{k=0}^{\infty}\left(x^{2}+2 D x\right)^{k} . \\
& \text { (x sufficiently small) }
\end{aligned}
$$

Choosing $i=n$, we obtain by comparison of coefficients of $x^{n}$

$$
A_{0}^{(n+2)}=\sum\left(\begin{array}{l}
y_{1}+y_{2} \\
y_{1}, y_{2}
\end{array}\right)(2 D)^{y_{2}} x^{2 y_{1}+y_{2}} \text {. }
$$

But $2 \mathrm{y}_{1}+\mathrm{y}_{2}=\mathrm{n}, \mathrm{y}_{1}+\mathrm{y}_{2}=\mathrm{n}-\mathrm{y}_{1}$,

$$
\begin{aligned}
A_{0}^{(n+2)}= & \sum\left(\begin{array}{c}
n-y_{I} \\
y_{I}
\end{array}\right)(2 D)^{n-2 y_{I}} \\
A_{0}^{(n+2)}= & \sum_{i=0}^{\left[\frac{n}{2}\right]}\left(\begin{array}{c}
n-i \\
i
\end{array}\right)(2 D)^{n-2 i} \\
& n=0,1, \ldots .
\end{aligned}
$$

We obtain from (3.1), for $n \Rightarrow 2 n-2$

$$
A_{0}^{(2 n)}=\sum_{i=0}^{n-1}\left(\begin{array}{c}
2 n-2-i \\
i
\end{array}\right)(2 D)^{2 n-2-2 i}, n=1,2, \ldots
$$

and for $n \Rightarrow 2 n-1$

$$
\left.\begin{array}{rl}
A_{0}^{(2 n+1)}= & \sum_{i=0}^{n-1}\left(\begin{array}{c}
2 n-1-i \\
i
\end{array}\right)(2 D)^{2 n-1-2 i} \\
& n=1,2, \ldots
\end{array}\right\}
$$

We shall verify formulas (3.2), (3.3), comparing the results with these from (2.8).

We obtain from the latter

$$
\begin{aligned}
& A_{O}^{(2)}=1, A_{O}^{(3)}=A_{O}^{(1)}+2 \mathrm{DA}_{O}^{(2)}=2 \mathrm{D}, \\
& A_{O}^{(4)}=A_{O}^{(2)}+2 \mathrm{DA}_{O}^{(3)}=1+4 \mathrm{D}^{2} ; \\
& A_{O}^{(5)}=A_{O}^{(3)}+2 \mathrm{DA}_{O}^{(4)}=4 \mathrm{D}+8 \mathrm{D}^{3} ; \\
& A_{O}^{(6)}=A_{O}^{(4)}+2 \mathrm{DA}_{O}^{(5)}=1+12 \mathrm{D}^{2}+16 \mathrm{D}^{4} .
\end{aligned}
$$

From (3.2) we obtain for $n=1,2,3$

$$
A_{O}^{(2)}=1, A_{O}^{(4)}=I+4 D^{2} ; A_{O}^{(6)}=1+12 D^{2}+16 D^{4} \text {. }
$$

From (3.3) we obtain for $\mathrm{n}=1,2,3$

$$
A_{0}^{(3)}=\sum_{i=0}^{0}\left(\begin{array}{c}
2 n-1-i \\
i
\end{array}\right) \quad(2 D)^{(2 n-1-2 i)}=2 D
$$




$$
\begin{aligned}
& A_{0}^{(5)}=\sum_{i=0}^{I}\left(\begin{array}{c}
2 n-1-i \\
i
\end{array}\right) \quad(2 D)^{(2 n-1-2 i)}=\left(\begin{array}{l}
2 \\
1
\end{array}\right) 2 D+(2 D)^{3} \\
& A_{O}^{(5)}=4 D+8 D^{3} . \\
& A_{0}^{(7)}=\sum_{i=0}^{2}\left(\begin{array}{c}
5-i \\
i
\end{array}\right) \quad(2 D)^{5-2 i}=\left(\begin{array}{l}
3 \\
2
\end{array}\right) 2 D+\left(\begin{array}{l}
4 \\
1
\end{array}\right)(2 D)^{3}+(2 D)^{5}, \\
& A_{0}^{(7)}=6 D+32 D^{3}+32 D^{5} .
\end{aligned}
$$

From (3.8) we obtain

$$
\begin{aligned}
A_{O}^{(7)} & =A_{O}^{(5)}+2 D A_{O}^{(6)}=4 D+8 D^{3}+2 D\left(1+12 D^{2}+16 D^{4}\right) \\
& =6 D+32 D^{3}+32 D^{5} .
\end{aligned}
$$

4. THE CONVERGENTS OF $\sqrt{2}$

We obtain from formula (1.7), since in our case $n=2$,

$$
\begin{aligned}
& a^{(0)}=a_{1}^{(0)}=\lim _{v \rightarrow \infty} \frac{A_{1}^{(v)}}{A_{0}^{(v)}}, \\
& w+D \simeq \frac{A_{1}^{(m+1)}}{A_{0}^{(m+1)}}, \quad m=1,2, \ldots
\end{aligned}
$$

With formula (2.11), we obtain from (4.1)

$$
\begin{aligned}
& w+D \approx \frac{A_{0}^{(m)}+2 D A_{O}^{(m+1)}}{A_{0}^{(m+1)}}=2 D+\frac{A_{0}^{(m)}}{A_{0}^{(m+1)}} \\
& w \simeq D+\frac{A_{O}^{(m)}}{A_{0}^{(m+1)}} .
\end{aligned}
$$

We investigate the special case $D=1$ and obtain from (4.3)

$$
\begin{aligned}
& \mathrm{m}=2, \sqrt{2} \approx 1+\frac{1}{2}=1.5 \\
& \mathrm{~m}=3, \sqrt{2} \approx 1+\frac{2}{5}=1.4 \\
& \mathrm{~m}=4, \sqrt{2} \approx 1+\frac{5}{12}=1.416 \\
& \mathrm{~m}=5, \sqrt{2} \approx 1+\frac{12}{29}=1.413 \\
& \mathrm{~m}=6, \sqrt{2} \approx 1+\frac{29}{70}=1.414
\end{aligned}
$$

Thus for $m=5,6$ we already obtain quite a tolerable approximation for $\sqrt{2}$.

As is known, $\sqrt{5}$ occupies an exceptional place in number theory. We set $D=2$, and obtain, with formula (3.6)

$$
\mathrm{w}=\sqrt{5} \approx 2+\frac{\mathrm{A}_{0}^{(\mathrm{m})}}{\mathrm{A}_{0}^{(\mathrm{m}+1)}} .
$$

We obtain from the previous calculations of the matricians with $D=2$ : 


$$
A_{O}^{(3)}=4, A_{O}^{(4)}=17, A_{O}^{(5)}=72, A_{O}^{(6)}=305, A_{O}^{(7)}=1294
$$

we obtain the approximation values

$$
\begin{aligned}
& \sqrt{5} \approx 2+\frac{4}{17}, \quad \approx \frac{17}{72}, \approx \frac{72}{305}, \approx \frac{305}{1294} \\
& \sqrt{5} \approx 2.235, \quad \approx 2.236, \approx 2.236 .
\end{aligned}
$$

Thus 2.236 is a good approximation of $\sqrt{5}$. That $\left(A_{0}^{(m)}, A_{0}^{(m+1)}\right)=1$ follows from (1.5).

5. THE CASE $n=3$

We denote again

$$
w=\sqrt[3]{D^{3}+1}
$$

and choose the fixed vector

$$
a^{(0)}=\left(w+2 D, w^{2}+D w+D^{2}\right) \text {. }
$$

With $a^{(0)}=\left(a_{1}^{(0)}(w), a_{2}^{(0)}(w)\right)$, we again apply the rule for calculating the components of the companion vectors

$$
b_{i}^{(v)}=a_{i}^{(v)}(D), i=1,2 ; v=0,1, \ldots
$$

We proceed with the GEA of $a^{(0)}$

$$
\begin{aligned}
b^{(0)} & =\left(D+2 D, D^{2}+D \cdot D+D^{2}\right) \\
b^{(0)} & =\left(3 D, 3 D^{2}\right) . \\
a^{(1)} & =(w+2 D-3 D)^{-1}\left(w^{2}+D w+D^{2}-3 D^{2}, I\right)= \\
& =(w-D)^{-1}\left(w^{2}+D w-2 D^{2}, I\right)= \\
& =(w-D)^{-1}((w-D)(w+2 D), 1), \\
a^{(1)} & =\left(w+2 D, w^{2}+D w+D^{2}\right)=a^{(0) .}
\end{aligned}
$$

By (5.4) the GEA of $a^{(0)}=\left((w+2), w^{2}+w D+D^{2}\right), w=\sqrt[3]{D^{3}+1}$ is purely periodic and the length of its primitive period $\ell=1$. We shall proceed to calculate the matricians of the GEA of this a $(0)$.

We have with $b^{(0)}=b^{(v)}=\left(3 D, 3 D^{2}\right), v=1,2, \ldots$

$$
\begin{aligned}
& \left.\begin{array}{c}
A_{0}^{(0)}=1, A_{O}^{(1)}=A_{0}^{(2)}=0, A_{0}^{(n+3)}=A_{0}^{(n)}+3 D A_{0}^{(n+1)}+3 D^{2} A_{0}^{(n+2)} \\
n=0,1, \ldots
\end{array}\right\} \\
& \sum_{n=0}^{\infty} A_{0}^{(n)} x^{n}=A_{0}^{(0)}+A_{0}^{(1)} x+A_{0}^{(2)} x^{2}+\sum_{n=0}^{\infty} A_{0}^{(n+3)} x^{n+3} \\
& =1+x^{3} \sum_{n=0}^{\infty} A_{0}^{(n+3)} x^{n}= \\
& =1+x^{3} \sum_{n=0}^{\infty}\left(A_{0}^{(n)}+3 D A_{0}^{(n+1)}+3 D^{2} A_{O}^{(n+2)}\right) x^{n}=
\end{aligned}
$$




$$
\begin{aligned}
& =1+x^{3} \sum_{n=0}^{\infty} A_{0}^{(n)} x^{n}+3 D x^{2} \sum_{n=0}^{\infty} A_{0}^{(n+1)} x^{n+1}+3 D^{2} x \sum_{n=0}^{\infty} A_{0}^{(n+2)} x^{n+2}= \\
& =1+x^{3} \sum_{n=0}^{\infty} A_{0}^{(n)} x^{n}+3 D x^{2}\left(-A_{0}^{(0)}+\sum_{n=0}^{\infty} A_{0}^{(n)} x^{n}\right)+ \\
& +3 D^{2} x\left(-A_{0}^{(0)}-A_{0}^{(1)} x+\sum_{n=0}^{\infty} A_{0}^{(n)} x^{n}\right)= \\
& \sum_{n=0}^{\infty}\left[1-\left(x^{3}+3 D x^{2}+3 D^{2} x\right) \sum_{n=0}^{\infty} A_{0}^{(n)} x^{n}-3 D x^{2}-3 D^{2} x\right. \\
& \sum_{n=0}^{\infty} A_{0}^{(n)} x^{n}=\frac{1-\left(3 D^{2} x+3 D x^{2}\right)}{\left.1-\left(3 x^{3}\right)\right] A_{0}^{(n)} x^{n}=1-3 D^{2} x-3 D x^{2}} \\
& \sum_{n=0}^{\infty} A_{0}^{(n)} x^{n}=1+\frac{\left.3 D x^{2}+x^{3}\right)}{1-\left(x^{3}+3 D x^{2}+3 D^{2} x\right)}
\end{aligned}
$$

and as before

$$
\sum_{n=0}^{\infty} A_{0}^{(n+3)} x^{n}=\sum_{k=0}^{\infty}\left(x^{3}+3 D x^{2}+3 D^{2} x\right)^{k}
$$

for sufficiently small $x$.

Comparing coefficients of $\mathrm{x}^{\mathrm{n}}$ on both sides of (5.6) we obtain

$$
\left.\begin{array}{c}
A_{O}^{(n+3)}=\sum_{3 y_{1}+2 y_{2}+y_{3}=n}\left(\begin{array}{l}
y_{1}+y_{2}+y_{3} \\
y_{1}, y_{2}, y_{3}
\end{array}\right)(3 D)^{y_{2}}\left(3 D^{2}\right)^{y_{3}} \\
A^{(n+3)}=\sum_{3 y_{1}+2 y_{2}+y_{3}=n}\left(\begin{array}{l}
y_{1}+y_{2}+y_{3} \\
y_{1}, y_{2}, y_{3}
\end{array}\right) 3^{y_{2}+y_{3}} D^{y_{2}+2 y_{3}} \\
n=0,1, \ldots ;\left(\begin{array}{l}
0 \\
0
\end{array}\right) \equiv 1 .
\end{array}\right\}
$$

From (5.7) the matricians are easily calculated, finding $y_{1}, y_{2}, y_{3}$ from the simple linear equations $3 \mathrm{y}_{1}+2 \mathrm{y}_{2}+\mathrm{y}_{3}=\mathrm{n}$. One proceeds in a lexiconographic order. We have

$$
\begin{array}{ll}
\mathrm{n}=0 ; & \mathrm{y}_{1}=\mathrm{y}_{2}=\mathrm{y}_{3}=0 ; \mathrm{A}_{0}^{(3)}=1 ; \\
\mathrm{n}=1 ; \mathrm{y}_{3}=1, \mathrm{y}_{1}=\mathrm{y}_{2}=0 ; \mathrm{A}_{0}^{(4)}=3 D^{2} ; \\
\mathrm{n}=2 ; \mathrm{y}_{1}=0=\mathrm{y}_{3}, \mathrm{y}_{2}=1 ; \mathrm{y}_{1}=\mathrm{y}_{2}=0, \mathrm{y}_{3}=2 ; \\
\mathrm{A}_{0}^{(5)}=3 \mathrm{D}+9 \mathrm{D}^{4} ; \\
\mathrm{n}=3 ; \mathrm{y}_{1}=1, \mathrm{y}_{2}=0, \mathrm{y}_{3}=0 ; \mathrm{y}_{1}=0, \mathrm{y}_{2}=1, \mathrm{y}_{3}=1 ; \\
\mathrm{y}_{1}=\mathrm{y}_{2}=0, \mathrm{y}_{3}=3 ; \\
\mathrm{A}_{0}^{(6)}=1+2 \cdot 3^{2} \mathrm{D}^{3}+3^{3} \mathrm{D}^{6}, \\
\mathrm{~A}_{0}^{(6)}=1+18 \mathrm{D}^{3}+27 \mathrm{D}^{6} .
\end{array}
$$




$$
\begin{aligned}
\mathrm{n}=4 ; & \mathrm{y}_{1}=1, \mathrm{y}_{2}=0, \mathrm{y}_{3}=1 ; \mathrm{y}_{1}=0, \mathrm{y}_{2}=2, \mathrm{y}_{3}=0 ; \\
& \mathrm{y}_{1}=0, \mathrm{y}_{2}=1, \mathrm{y}_{3}=2 ; \mathrm{y}_{1}=\mathrm{y}_{2}=0, \mathrm{y}_{3}=4 ; \\
& \mathrm{A}_{0}^{(7)}=2 \cdot 3 D^{2}+1.3^{2} D^{2}+\left(\begin{array}{l}
3 \\
2
\end{array}\right) \cdot 3 D\left(3 D^{2}\right)^{2}+3^{4} D^{8} \\
& \mathrm{~A}_{0}^{(7)}=15 D^{2}+81 D^{5}+81 D^{8} .
\end{aligned}
$$

One could verify these results by means of formula (5.5). From this we obtain

$$
\begin{aligned}
A_{O}^{(8)}= & A_{O}^{(5)}+3 D A_{O}^{(6)}+3 D^{2} A_{O}^{(7)}=3 D+9 D^{4}+ \\
& +\left(1+18 D^{3}+27 D^{6}\right) 3 D+3 D^{2}\left(15 D^{2}+81 D^{5}+81 D^{8}\right)= \\
= & 6 D+108 D^{4}+324 D^{7}+243 D^{10} . \\
A_{O}^{(9)}= & A_{O}^{(6)}+3 D A_{0}^{(7)}+3 D^{2} A_{O}^{(8)}= \\
= & 1+18 D^{3}+27 D^{6}+3 D\left(15 D^{2}+81 D^{5}+81 D^{8}\right)+ \\
& +3 D^{2}\left(6 D+108 D^{4}+324 D^{7}+243 D^{10}\right) \\
A_{O}^{(9)}= & 1+81 D^{3}+594 D^{6}+1215 D^{9}+729 D^{12} .
\end{aligned}
$$

It would be an interesting problem whether calculating preference should be given to formula (5.5) or (5.7).

We shall shortly discuss the number of solutions of $3 \mathrm{y}_{1}+2 \mathrm{y}_{2}+\mathrm{y}_{3}=3 \mathrm{n}$

$$
\begin{array}{llll}
\mathrm{y}_{1}=\mathrm{n} ; & \mathrm{y}_{2}=\mathrm{y}_{3}=0 ; & 1 \text { solution } & \\
\mathrm{y}_{1}=\mathrm{n}-1 ; & \mathrm{y}_{2}=1, \mathrm{y}_{3}=1 ; & \mathrm{y}_{2}=0, \mathrm{y}_{3}=3 ; & 2 \text { solutions } \\
\mathrm{y}_{1}=\mathrm{n}-2 ; & \mathrm{y}_{2}=3, \mathrm{y}_{3}=0 ; & \mathrm{y}_{2}=2, \mathrm{y}_{3}=2 ; & \\
& \mathrm{y}_{2}=1, \mathrm{y}_{3}=4 ; & \mathrm{y}_{2}=0, \mathrm{y}_{3}=6 ; & 4 \text { solutions } \\
\mathrm{y}_{1}=\mathrm{n}-3 ; & \mathrm{y}_{2}=4, \mathrm{y}_{3}=1 ; & \mathrm{y}_{2}=3, \mathrm{y}_{3}=3 ; & \\
& \mathrm{y}_{2}=2, \mathrm{y}_{3}=5 ; & \mathrm{y}_{2}=1, \mathrm{y}_{3}=7 ; & \\
& \mathrm{y}_{2}=0, \mathrm{y}_{3}=9 ; & &
\end{array}
$$

Thus the number of solutions of $3 y_{1}+2 y_{2}+y_{3}=3 n$ equals

$$
\begin{aligned}
S_{3 n} & =1+(2+4)+(5+7)+(8+10)+(11+13)+\cdots \quad \text { (n numbers) } \\
S_{3 n} & =(1+4+7+\cdots)+(2+5+8+11+\cdots) \\
& \approx\left(\left[2+\left(\left[\frac{n}{2}\right]-1\right) 3\right]+\left[2 \cdot 2+\left(\left[\frac{n}{2}\right]-1\right) 3\right]\right)\left[\frac{n}{2}\right] \\
& \approx\left[\frac{3 n^{2}}{2}\right] .
\end{aligned}
$$

Thus, approximately

$$
S_{3 n} \simeq\left[\frac{3 n^{2}}{2}\right]
$$

For $n=2, s_{6}=7 \approx \frac{3.2^{2}}{2}=6$.

From the other side, $A_{0}^{(3 n)}$ contains exactly $2(n-1)+1=2 n-1$ summands, as the reader can easily verify. 
In order to calculate $A_{O}^{(3 n)}$ by formula (5.5), one has to calculate the preceding $3 n$ matricians $A_{0}^{(0)}, A_{0}^{(1)}, \ldots, A_{0}^{(3 n-1)}$, so the number of these manipulations equals $3 n(2 n-1)$. The author therefore conjectures, that it is preferable to calculate the matricians, from a time and operation saving profit view, by formula (5.7).

We shall now calculate the matricians $A_{1}^{(v)}$ and $A_{2}^{(v)}$, expressing them as linear functions of $A_{0}^{(t)}$. We have, with a $(1)^{2}=a^{(0)}=$ $\left(w+2 D, w^{2}+D w+D^{2}\right)$, and in virtue of formula (1.6)

$$
\left.\begin{array}{c}
w+2 D=\frac{A_{1}^{(n)}+(w+2 D) A_{1}^{(n+1)}+\left(w^{2}+D w+D^{2}\right) A_{1}^{(n+2)}}{A_{0}^{(n)}+(w+2 D) A_{0}^{(n+1)}+\left(w^{2}+D w+D^{2}\right) A_{0}^{(n+2)}} \\
(w+2 D) A_{0}^{(n)}+(w+D) A_{0}^{(n+1)}+\left(w^{2}+D w+D^{2}\right) A_{0}^{(n+2)}= \\
A_{1}^{(n)+(w+D) A_{1}^{(n+1)}+\left(w^{2}+D w+D^{2}\right) A_{1}^{(n+2)} .}
\end{array}\right\}
$$

Comparing the coefficients of the powers of the irrational $w^{2}$ on both sides of (5.9), we obtain

$$
A_{1}^{(n+2)}=A_{0}^{(n+1)}+3 D A_{0}^{(n+2)} .
$$

In the same way we obtain from

$$
\begin{gathered}
\left(w^{2}+D w+D^{2}\right)\left(A_{0}^{(n)}+(w+2 D) A_{0}^{(n+1)}+\left(w^{2}+D w+D^{2}\right) A_{0}^{(n+2)}\right)= \\
=A_{2}^{(n)}+(w+2 D) A_{2}^{(n+1)}+\left(w^{2}+D w+D^{2}\right) A_{2}^{(n+2)} \\
A_{2}^{(n+2)}=A_{0}^{(n)}+3 D A_{0}^{(n+1)}+3 D^{2} A_{0}^{(n+2)} .
\end{gathered}
$$

6. THE CONVERGENTS OF $\sqrt[3]{D^{3}+1}$

We obtain in case $n=3$ with $w=\sqrt[3]{D^{3}+1}$,

$$
a_{1}^{(0)}=w+2 D, a_{2}^{(0)}=w^{2}+D w+D^{2} \text {, }
$$

and by formulas $(1.7),(5.10),(5.11)$

$$
\begin{aligned}
& w+2 D=\lim _{n \rightarrow \infty} \frac{A_{1}^{(n+1)}}{A_{0}^{(n+1)}}=\lim _{n \rightarrow \infty} \frac{A_{0}^{(n)}+3 D A_{0}^{(n+1)}}{A_{0}^{(n+1)}}= \\
& =3 D+\lim _{n \rightarrow \infty} \frac{A_{0}^{(n)}}{A_{0}^{(n+1)}} . \\
& w=D+\lim _{n \rightarrow \infty} \frac{A_{0}^{(n)}}{A_{0}^{(n+1)}} .
\end{aligned}
$$

Substituting in (6.1) the values for the matricians $A_{O}^{(v)}$ from (5.7), we obtain with $\mathrm{n} \Rightarrow \mathrm{n}+3$ 


$$
\mathrm{w}=\mathrm{D}+\lim _{\mathrm{n} \rightarrow \infty} \frac{\sum_{3 \mathrm{y}_{1}+2 \mathrm{y}_{2}+\mathrm{y}_{3}=\mathrm{n}}\left(\begin{array}{l}
\mathrm{y}_{1}+\mathrm{y}_{2}+\mathrm{y}_{3} \\
\mathrm{y}_{1}, \mathrm{y}_{2}, \mathrm{y}_{3}
\end{array}\right) 3^{\mathrm{y}_{2}+\mathrm{y}_{3} \mathrm{D}_{2}+2 \mathrm{y}_{3}}}{\sum_{3 \mathrm{y}_{1}+2 \mathrm{y}_{2}+\mathrm{y}_{3}=\mathrm{n}+1}\left(\begin{array}{l}
\mathrm{y}_{1}+\mathrm{y}_{2}+\mathrm{y}_{3} \\
\mathrm{y}_{1}, \mathrm{y}_{2}, \mathrm{y}_{3}
\end{array}\right)_{3} \mathrm{y}_{2}+\mathrm{y}_{3} \mathrm{y}_{2}+2 \mathrm{y}_{3}}
$$

(6.2) is a very interesting and simple formula for calculating the convergents of $\sqrt[3]{\mathrm{D}^{3}+1}$. We further obtain by the same method

$$
\begin{aligned}
& w^{2}+D w+D^{2}=\lim _{n \rightarrow \infty} \frac{A_{2}^{(n+2)}}{A_{0}^{(n+2)}}= \\
& =\lim _{n \rightarrow \infty} \frac{A_{0}^{(n)}+3 D A_{0}^{(n+1)}+3 D^{2} A_{0}^{(n+2)}}{A_{0}^{(n+2)}} \\
& =3 D^{2}+\lim _{n \rightarrow \infty} \frac{A_{0}^{(n)}}{A_{0}^{(n+2)}}+3 D \lim _{n \rightarrow \infty} \frac{A_{0}^{(n+1)}}{A_{0}^{(n+2)}} ; \\
& w^{2}+D\left(D+\lim _{n \rightarrow \infty} \frac{A_{O}^{(n+1)}}{A_{O}^{(n+2)}}\right)= \\
& =2 D^{2}+\lim _{n \rightarrow \infty} \frac{A_{0}^{(n)}}{A_{0}^{(n+2)}}+3 D \lim _{n \rightarrow \infty} \frac{A_{0}^{(n+1)}}{A_{0}^{(n+2)}}, \\
& w^{2}=D^{2}+\lim _{n \rightarrow \infty} \frac{A_{0}^{(n)}}{A_{0}^{(n+2)}}+2 D \lim _{n \rightarrow \infty} \frac{A_{0}^{(n+1)}}{A_{0}^{(n+2)}} \\
& w^{2}=D^{2}+\lim _{n \rightarrow \infty} \mid \frac{\sum_{1}+2 y_{2}+y_{3}=n-3}{\sum_{3 y_{1}+2 y_{2}+y_{3}=n-1}\left(\begin{array}{l}
y_{1}+y_{2}+y_{3} \\
y_{1}, y_{2}, y_{3}
\end{array}\right) 3^{y_{2}+y_{3}} D^{y_{2}+2 y_{3}}}
\end{aligned}
$$

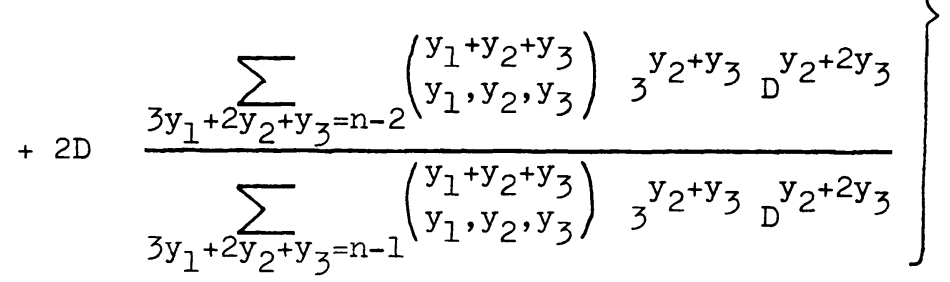

This limiting expression for $\mathrm{w}^{2}$ takes on a special simple form for $\mathrm{D}=1$. For $\mathrm{D}=1$ the matricians, which were calculated previously, became $A_{O}^{(3)}=1, A_{O}^{(4)}=3, A_{O}^{(5)}=12, A_{O}^{(6)}=46, A_{O}^{(7)}=177$, $A_{0}^{(8)}=681, A_{0}^{(9)}=2620$. 
With those values we obtain from the formula (5.11)

$$
\begin{aligned}
& \sqrt[3]{2}=1+\lim _{n \rightarrow \infty} \frac{A_{0}^{(n)}}{A_{0}^{(n+1)}}, \\
& \sqrt[3]{2} \approx 1+\frac{1}{3}, 1+\frac{3}{12}, 1+\frac{12}{46}, 1+\frac{46}{177}, 1+\frac{177}{681}, 1+\frac{681}{2620}, \\
& \sqrt[3]{2} \approx 1.3,1.25,1.26,1.26,1.26,1.26 .
\end{aligned}
$$

Thus $\sqrt[3]{2}=1.26$ is quite an exact approximation. We have

$$
2 \approx 1.26^{3}=2.000376 \text {. }
$$

We further obtain from the first line of (6.3) with $D=1$

$$
\begin{aligned}
\sqrt[3]{4} \approx 1+\frac{A_{0}^{(n)}}{A_{0}^{(n+2)}}+\frac{2 A_{0}^{(n+1)}}{A_{0}^{(n+2)}}, \\
\sqrt[3]{4} \approx 1+\frac{1}{12}+\frac{1}{2}, 1+\frac{3}{16}+\frac{24}{46}, 1+\frac{12}{177}+\frac{92}{177}, \\
1+\frac{46}{681}+\frac{354}{681}, 1+\frac{177}{2620}+\frac{1362}{2620} .
\end{aligned}
$$

$\sqrt[3]{4}=1.58,1.59,1.59,1.59,1.58$. Thus $\sqrt[3]{4}=1.59$ is a satisfying approximation.

For $D=2,3 w^{2}=\sqrt[3]{81}=3 \sqrt[3]{3}$.

Thus also $\sqrt{3}$ can be easily and perfectly approximated by formula (5.11).

$$
\begin{array}{r}
\text { 7. THE CASE } \mathrm{n}=5 \\
\text { We denote again } \\
w=\sqrt[5]{\mathrm{D}^{5}+1}
\end{array}
$$

and choose the fixed vector

$$
\begin{aligned}
a^{(0)=} & \left(w+4 D, w^{2}+3 w D+6 D^{2}, w^{3}+2 w^{2} D+3 w D^{2}+4 D^{3},\right. \\
& \left.w^{4}+D w^{3}+D^{2} w^{2}+D^{3} w+D^{4}\right) .
\end{aligned}
$$

With $a^{(0)}=\left(a_{1}^{(0)}(w), a_{2}^{(0)}(w), a_{3}^{(0)}(w), a_{4}^{(0)}(w)\right)$, we again apply the rule for calculating the components of the companion vectors $b_{i}^{(v)}=a_{i}^{(v)}(D), i=1,2,3,4 ; v=0,1, \ldots$.

We proceed with the GEA of $\mathrm{a}^{(\mathrm{O})}$ and have

$$
\begin{aligned}
b^{(0)}= & \left(D+4 D, D^{2}+3 D^{2}+6 D^{2}, D^{3}+2 D^{3}+3 D^{3}+4 D^{3}, D^{4}+D^{4}+D^{4}+D^{4}+D^{4}\right) \\
b^{(0)}= & \left(5 D, 10 D^{2}, 10 D^{3}, 5 D^{4}\right) \\
b^{(0)}= & \left(\left(\begin{array}{l}
5 \\
1
\end{array}\right) D,\left(\begin{array}{l}
5 \\
2
\end{array}\right) D^{2},\left(\begin{array}{l}
5 \\
3
\end{array}\right) D^{3},\left(\begin{array}{l}
5 \\
4
\end{array}\right) D^{4}\right) . \\
a^{(1)}= & (w+4 D-5 D)^{-1} \cdot\left(w^{2}+3 w D+6 D^{2}-10 D^{2},\right. \\
& \left.w^{3}+2 w^{2} D+3 w D^{2}+4 D^{3}-10 D^{3}, w^{4}+D w^{3}+D^{2} w^{2}+D^{3} w+D^{4}-5 D^{4}, I\right), \\
a^{(1)}= & (w-D)^{-1} \cdot\left((w-D)(w+4 D),(w-D)\left(w^{2}+3 w D+6 D^{2}\right),\right. \\
& (w-D)\left(w^{3}+2 w^{2} D+3 w D^{2}+4 D^{3},(w-D)\left(w^{4}+w^{3} D+w^{2} D^{2}+w D^{3}+D^{4}\right)\right), \\
a^{(1)}= & a^{(0) .}
\end{aligned}
$$


Since $(w-D)\left(w^{3}+w^{3} D+w^{2} D^{2}+w D^{3}+D^{4}\right)=w^{5}-D^{5}=1$. Thus the GEA of a ${ }^{(0)}$ is purely periodic with lengths of primitive period $\ell=1$. We shall calculate the matricians of ${ }^{(0)}$.

With (3.6) we have

$$
\begin{aligned}
& A_{O}^{(0)}=1, A_{O}^{(1)}=A_{O}^{(2)}=A_{0}^{(3)}=A_{0}^{(4)}=0 . \\
& A_{O}^{(n+5)}=A_{O}^{(n)}+5 D A_{O}^{(n+1)}+10 D^{2} A_{O}^{(n+2)}+10 D^{3} A_{O}^{(n+3)}+5 D^{4} A_{O}^{(n+4)},\left\{\begin{array}{l}
(7.5) \\
n=0,1, \ldots
\end{array}\right.
\end{aligned}
$$

Proceeding as in cases $n=2,3$ using Euler's functions, we obtain

$$
\begin{aligned}
& \sum_{n=0}^{\infty} A_{0}^{(n)} x^{n}=1+\sum_{n=0}^{\infty} A_{0}^{(n+5)} x^{n+5}= \\
& =1+\sum_{n=0}^{\infty}\left(A_{0}^{(n)}+5 D A_{0}^{(n+1)}+10 D^{2} A_{0}^{(n+2)}+10 D^{3} A_{0}^{(n+3)}+5 D^{4} A_{0}^{(n+4)}\right) \cdot x^{n+5} \\
& =x^{5} \sum_{n=0}^{\infty} A_{0}^{(n)} x^{n}+5 D\left(-1+\sum_{n=0}^{\infty} A_{0}^{(n)} x^{n}\right) x^{4}+ \\
& +10 D^{3} x^{3}\left(-1+\sum_{n=0}^{\infty} A_{0}^{(n)} x^{n}\right)+10 D^{3} x^{2}\left(-1+\sum_{n=0}^{\infty} A_{0}^{(n)} x^{n}\right)+ \\
& +5 D^{4} x\left(-1+\sum_{n=0}^{\infty} A(n) x^{n}\right) \\
& {\left[1-\left(x^{5}+5 D x^{4}+10 D^{2} x^{3}+10 D^{3} x^{2}+5 D^{4} x\right)\right] \sum_{n=0}^{\infty} A(n) x^{n}=} \\
& =-\left(5 D x^{4}+10 D^{2} x^{3}+10 D^{3} x^{2}+5 D^{4} x\right), \\
& \sum_{n=0}^{\infty} A_{0}^{(n+5)} x^{n}=\frac{1}{1-\left(x^{5}+5 D x^{4}+10 D^{2} x^{3}+10 D^{3} x^{2}+5 D^{4} x\right)} \\
& =\sum_{k=0}^{\infty}\left(x^{5}+5 D x^{4}+10 D^{2} x^{3}+10 D^{3} x^{2}+5 D^{4} x\right) \text {. }
\end{aligned}
$$

Hence

$$
\begin{aligned}
& A_{0}^{(n+5)}=\sum_{5 y_{1}+4 y_{2}+3 y_{3}+2 y_{4}+y_{5}=n}\left(\begin{array}{l}
y_{1}+y_{2}+y_{3}+y_{4}+y_{5} \\
y_{1}, y_{2}, y_{3}, y_{4}, y_{5}
\end{array}\right)(5 D)^{y_{2}}\left(10 D^{2}\right)^{y_{3}}\left(10 D^{3}\right)^{y_{4}}\left(5 D^{4}\right)^{y_{5}} \\
& =\sum_{5 y_{1}+4 y_{2}+3 y_{3}+2 y_{4}+y_{5}=n}\left(\begin{array}{l}
y_{1}+y_{2}+y_{3}+y_{4}+y_{5} \\
y_{1}, y_{2}, y_{3}, y_{4}, y_{5}
\end{array}\right)_{5} y_{2}+y_{3}+y_{4}+y_{5} y_{3}+y_{4} y_{2}+2 y_{3}+3 y_{4}+4 y_{5}
\end{aligned}
$$




$$
\left.\begin{array}{c}
A_{0}^{(n+5)}=\sum_{\sum_{i=0}^{4}(5-i) y_{i+1}=n}\left(\begin{array}{l}
\sum_{k=1}^{5} y_{k} \\
y_{1}, y_{2}, y_{3}, y_{4}, y_{5}
\end{array}\right) \sum^{4}{ }_{k=1}^{4} y_{k+1} y_{3}+y_{4} y_{2}+2 y_{3}+3 y_{4}+4 y_{5}
\end{array}\right\}
$$

Formula (7.6) is, indeed, a bit frightening, but it calculates explicitly any $A_{O}^{(n+5)}(n=0,1, \ldots)$ just by solving the linear equations $\sum_{i=0}^{4}(5-i) y_{i+1}=n$.

We shall calculate a few matricians from formula (7.6). When one of the $y_{i}-s$ will not be mentioned, its value is understood to equal zero.

$$
\begin{aligned}
& \mathrm{n}=1 ; \mathrm{y}_{5}=1, \mathrm{~A}_{0}^{(6)}=5 \mathrm{D}^{4} \text {; } \\
& \mathrm{n}=2 \text {; i) } \mathrm{y}_{4}=1 \text {; ii) } \mathrm{y}_{5}=2 ; \mathrm{A}_{0}^{(7)}=10 \mathrm{D}^{3}+25 \mathrm{D}^{8} \text {; } \\
& \mathrm{n}=3 \text {; i) } \mathrm{y}_{3}=1 \text {; ii) } \mathrm{y}_{4}=\mathrm{y}_{5}=1 \text {; iii) } \mathrm{y}_{5}=3 \text {, } \\
& A_{0}^{(8)}=10 D^{2}+100 D^{7}+125 D^{12} \text {; } \\
& \mathrm{n}=4 \text {; i) } \mathrm{y}_{2}=1 \text {; ii) } \mathrm{y}_{3}=\mathrm{y}_{5}=1 \text {; iii) } \mathrm{y}_{4}=2 \text {; iv) } \mathrm{y}_{4}=1, \mathrm{y}_{5}=2 \text {; } \\
& \text { v) } y_{5}=4, A_{0}^{(9)}=5 D+100 D^{6}+100 D^{6}+750 D^{11}+625 D^{16} \text {, } \\
& A_{0}^{(9)}=5 D+200 D^{6}+750 D^{11}+625 D^{16} \text {; } \\
& \mathrm{n}=5 \text {; i) } \mathrm{y}_{1}=1 \text {; ii) } \mathrm{y}_{2}=\mathrm{y}_{3}=1 \text {; iii) } \mathrm{y}_{3}=\mathrm{y}_{4}=1 \text {; iv) } \mathrm{y}_{3}=1, \mathrm{y}_{5}=2 \text {; } \\
& \text { v) } \mathrm{y}_{4}=2, \mathrm{y}_{5}=1 \text {; vi) } \mathrm{y}_{4}=1, \mathrm{y}_{5}=3 \text {; vii) } \mathrm{y}_{5}=5 \text {, } \\
& A_{O}^{(10)}=1+50 D^{5}+200 D^{5}+750 D^{10}+1500 D^{10}+5000 D^{15}+3125 D^{20} \text {. } \\
& A_{0}^{(10)}=1+250 D^{5}+2250 D^{10}+5000 D^{15}+3125 D^{20} \text {. }
\end{aligned}
$$

We shall further calculate the matricians $A_{i}^{(v)}, i=1,2,3,4$. We have

$$
\begin{aligned}
A_{1}^{(n)} & +(w+4 D) A_{I}^{(n+1)}+\left(w^{2}+3 D w+6 D^{2}\right) A_{1}^{(n+2)}+ \\
& +\left(w^{3}+2 w^{2} D+3 w D^{2}+4 D^{3}\right) A_{1}^{(n+3)}+ \\
& +\left(w^{4}+D w^{3}+D^{2} w^{2}+D^{3} w+D^{4}\right) A_{1}^{(n+4)}= \\
& =(w+4 D) A_{0}^{(n)}+(w+4 D) A_{0}^{(n+1)}+\left(w^{2}+3 D w+6 D^{2}\right) A_{0}^{(n+2)}+ \\
& +\left(w^{3}+2 w^{2} D+3 w D^{2}+4 D^{3}\right) A_{0}^{(n+3)}+ \\
& +\left(w^{4}+D w^{3}+D^{2} w^{2}+D^{3} w+D^{4}\right) A_{0}^{(n+4)} .
\end{aligned}
$$

Comparing powers of $w^{4}$ on both sides of (7.7) we obtained

$$
A_{1}^{(n+4)}=A_{0}^{(n+3)}+5 D A_{0}^{(n+4)} .
$$


Further

$$
\begin{aligned}
A_{2}^{(n)}+ & (w+4 D) A_{2}^{(n+1)}+\cdots+\left(w^{4}+\cdots+D^{4}\right) A_{2}^{(n+4)}= \\
= & \left(w^{2}+3 D w+6 D^{2}\right)\left[A_{0}^{(n)}+\cdots+\left(w^{2}+3 D w+6 D^{2}\right) A_{0}^{(n+2)}\right. \\
& \left.+\left(w^{3}+2 w^{2} D+3 w D^{2}+4 D^{3}\right) A_{0}^{(n+3)}+\left(w^{4}+D w^{3}+D^{2} w^{2}+D^{3} w+D^{4}\right) A_{0}^{(n+4)}\right] . \\
& A_{2}^{(n+4)}=A_{0}^{(n+2)}+5 D A_{O}^{(n+3)}+10 D^{2} A_{O}^{(n+4)}
\end{aligned}
$$

We leave it to the reader to verify, by the same method the results

$$
\begin{aligned}
& A_{3}^{(n+4)}=A_{O}^{(n+1)}+5 D A_{O}^{(n+2)}+10 D^{2} A_{O}^{(n+3)}+10 D^{3} A_{O}^{(n+4)} \\
& A_{4}^{(n+4)}=A_{0}^{(n)}+5 D A_{0}^{(n+1)}+10 D^{2} A_{O}^{(n+2)}+10 D^{3} A_{O}^{(n+3)}+5 D^{4} A_{O}^{(n+4)} .
\end{aligned}
$$

We thus have obtained

$$
\begin{aligned}
& A_{1}^{(n+4)}=A_{0}^{(n+3)}+\left(\begin{array}{l}
5 \\
1
\end{array}\right) A_{0}^{(n+4)} \\
& A_{2}^{(n+4)}=A_{0}^{(n+2)}+\left(\begin{array}{l}
5 \\
1
\end{array}\right) A_{0}^{(n+3)}+\left(\begin{array}{l}
5 \\
2
\end{array}\right) A_{0}^{(n+4)} \\
& A_{3}^{(n+4)}=A_{0}^{(n+1)}+\left(\begin{array}{l}
5 \\
1
\end{array}\right) D A_{0}^{(n+2)}+\left(\begin{array}{l}
5 \\
2
\end{array}\right) D^{2} A_{0}^{(n+3)}+\left(\begin{array}{l}
5 \\
3
\end{array}\right) D^{3} A_{O}^{(n+4)} \\
& A_{4}^{(n+4)}=A_{0}^{(n)}+\left(\begin{array}{l}
5 \\
1
\end{array}\right) D A_{0}^{(n+1)}+\left(\begin{array}{l}
5 \\
2
\end{array}\right) D^{2} A_{0}^{(n+2)}+\left(\begin{array}{l}
5 \\
3
\end{array}\right) D^{3} A_{O}^{(n+3)}+\left(\begin{array}{l}
5 \\
4
\end{array}\right) D^{4} A_{O}^{(n+4)} \\
& A_{i}^{(n+4)}=\sum_{j=0}^{i} A_{0}^{(n+4-j)}\left(\begin{array}{c}
5 \\
i-j
\end{array}\right) D^{i-j}, \quad i=1,2,3,4, n=0,1, \ldots
\end{aligned}
$$

To conclude with this first paper of the sequence, we shall still approximate the number $\sqrt[5]{2}, \sqrt[5]{4}$. From (7.1) we have, with $D=1$, $\mathbf{w}=\sqrt[5]{2}$.

From (7.2) we obtain, with Theorem I,

$$
w+4=\lim _{n \rightarrow \infty} \frac{A_{1}^{(n+6)}}{A_{0}^{(n+6)}}
$$

and from $(7.8)$

$$
\begin{aligned}
& w+4=\lim _{n \rightarrow \infty} \frac{A_{0}^{(n+5)}+5 A_{0}^{(n+6)}}{A_{0}^{(n+6)}}, \\
& w=1+\lim _{n \rightarrow \infty} \frac{A_{0}^{(n+5)}}{A_{0}^{(n+6)}} .
\end{aligned}
$$

From (7.14) and (7.6) we finally obtain 
5

$\sqrt{2} \approx 1+$

$\sum_{5 y_{1}+4 y_{2}+3 y_{3}+2 y_{4}+y_{5}=n}\left(\begin{array}{l}y_{1}+y_{2}+y_{3}+y_{4}+y_{5} \\ y_{1}, y_{2}, y_{3}, y_{4}, y_{5}\end{array}\right)_{5}\left(y_{2}+y_{3}+y_{4}+y_{5}\right)_{2}\left(y_{3}+y_{4}\right)_{D}\left(y_{2}+2 y_{3}+3 y_{4}+4 y_{5}\right)$

$\sum_{5 y_{1}+4 y_{2}+3 y_{3}+3 y_{4}+y_{5}=n+1}\left(\begin{array}{l}y_{1}+y_{2}+y_{3}+y_{4}+y_{5} \\ y_{1}, y_{2}, y_{3}, y_{4}, y_{5}\end{array}\right)_{5}\left(y_{2}+y_{3}+y_{4}+y_{5}\right)_{2}\left(y_{3}+y_{4}\right)_{D}\left(y_{2}+2 y_{3}+3 y_{4}+4 y_{5}\right)$

Substituting in (7.14) the values for $\mathrm{n}=1,2,3,4$ we obtain

$$
\begin{aligned}
& w=\sqrt[5]{2} \approx 1+\frac{5}{35}, 1+\frac{35}{235} 1+\frac{235}{1580}, 1+\frac{1580}{10626}, \\
& \sqrt[5]{2} \approx 1.143,1.149,1.149,1.149 .
\end{aligned}
$$

It seems that 1.149 is a good approximation of $\sqrt[5]{2}$. Indeed,

$$
1.149^{5} \approx 2 .
$$

From (7.2) and Theorem 1 we further obtain, in virtue of (7.9)

$$
\begin{aligned}
& w^{2}+3 w D+6 D^{2}=\lim _{n \rightarrow \infty} \frac{A_{2}^{(n+4)}}{A_{0}^{(n+4)}}=10 D^{2}+5 D \lim _{n \rightarrow \infty} \frac{A_{0}^{(n+3)}}{A_{0}^{(n+4)}}+\lim _{n \rightarrow \infty} \frac{A_{0}^{(n+2)}}{A_{0}^{(n+4)}}, \\
& w^{2}+3 D\left(D+\lim _{n \rightarrow \infty} \frac{A_{0}^{(n+3)}}{A_{0}^{(n+4)}}\right)+6 D^{2}=10 D^{2}+5 D \lim _{n \rightarrow \infty} \frac{A_{0}^{(n+3)}}{A_{0}^{(n+4)}}+\lim _{n \rightarrow \infty} \frac{A_{0}^{(n+2)}}{A_{0}^{(n+4)}},
\end{aligned}
$$

Hence

$$
w^{2}=D^{2}+2 D \lim _{n \rightarrow \infty} \frac{A_{0}^{(n+3)}}{A_{0}^{(n+4)}}+\lim _{n \rightarrow \infty} \frac{A_{0}^{(n+2)}}{A_{0}^{(n+4)}}
$$

with $D=1$, and the approximate value of $w$, we obtain

$$
\begin{aligned}
& w^{2} \approx 1+2 \cdot 0.149+\frac{A_{0}^{(n+2)}}{A_{0}^{(n+4)}} \\
& w^{2} \approx 1.298+\frac{A_{0}^{(n+2)}}{A_{0}^{(n+4)}} \cdot
\end{aligned}
$$

From here $\mathrm{w}^{2}$ can be easily evaluated exact to two places.

\section{REFERENCES}

1. JACOBI, C.G.J. Allgemeine Theorie der kettenbruchaehnlichen Algorithmen, in velchen jede $\mathrm{Zahl}$ aus drei vorhergehenden gebildet wird, J.f.d. reine angew. Math. 69 (1969), 29-64.

2. PERRON, 0 . Grundlagen fler eine Theorie des Jacobischen Kettenbruchalgorithmus, Math. Ann. 64 (1907), 1-76. 
3. BAICA, M. An algorithm in a complex field and its application to the calculation of units. Pacific J. Math. Vol. 110. No. 1 (1984), 21-40.

4. BERNSTEIN, L. The Jacobi-Perron algorithm, its theory and applications. Springer, Berlin-Heidelberg-New York. Lect. Notes. Math. $207^{\text {(1971). }}$

5. PERRON, O. Ein neues Konvergenzkriterium fur Jacobi-Ketten 2. Ordnung. Arch. Math. Phys. (Reihe 3) 17 (1911), 204-211.

6. BERNSTEIN, L. Representation of $\sqrt[n]{D^{n}-d}$ as a periodic continued fraction by Jacobi's algorithm. Math. Nachr. 29 (1965), 179-200.

7. CARLITZ, L. Some combinatorial identities of Bernstein, SIAM J. Math. Anal. 9 (1978), 65-75.

8. CARLITZ, L. Recurrences of the third order and related combinatorial identities, Fibonacci Quart. 16 (1978), 11-18.

9. HERMITE, CH. Letter to C.G.J. Jacobi, J.f.d. reine angew. Math. 40 (1939), 286. 


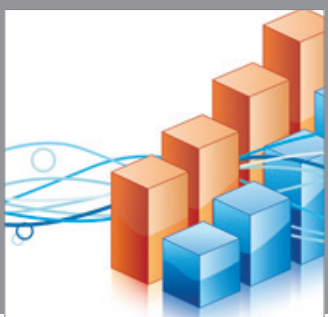

Advances in

Operations Research

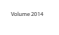

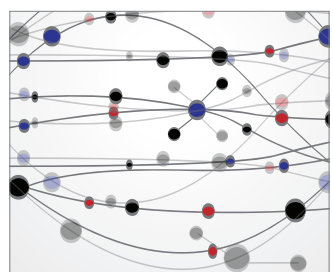

\section{The Scientific} World Journal
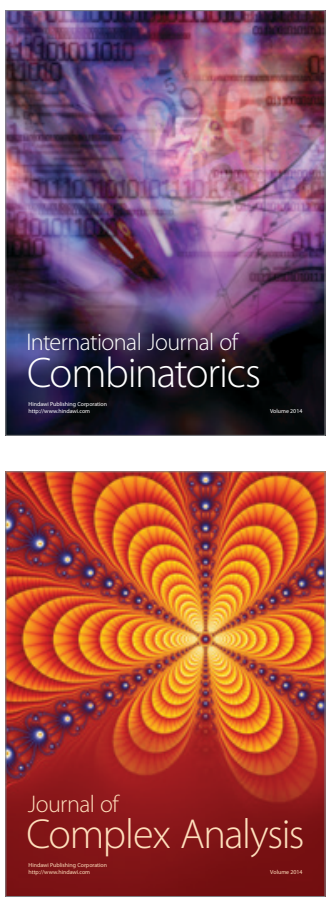

International Journal of

Mathematics and

Mathematical

Sciences
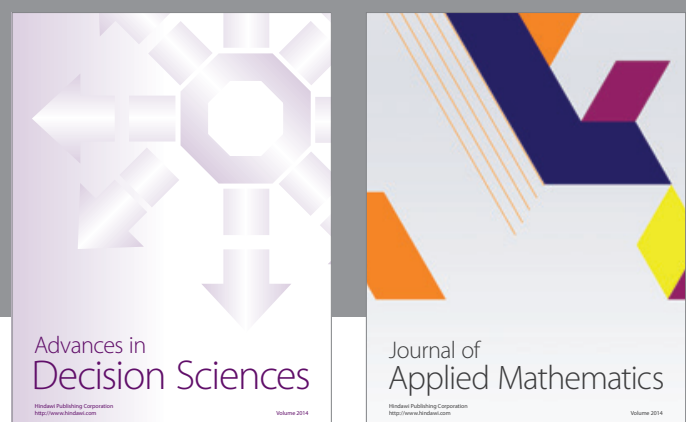

Journal of

Applied Mathematics
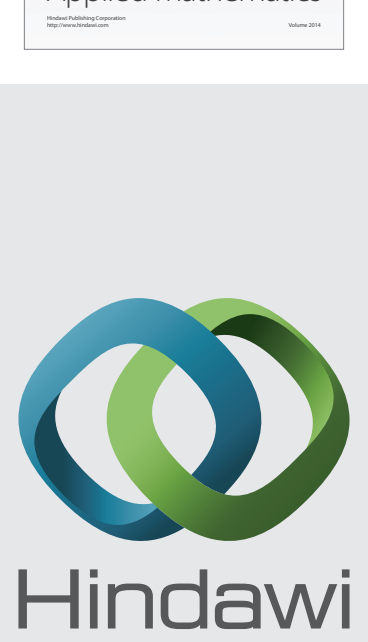

Submit your manuscripts at http://www.hindawi.com
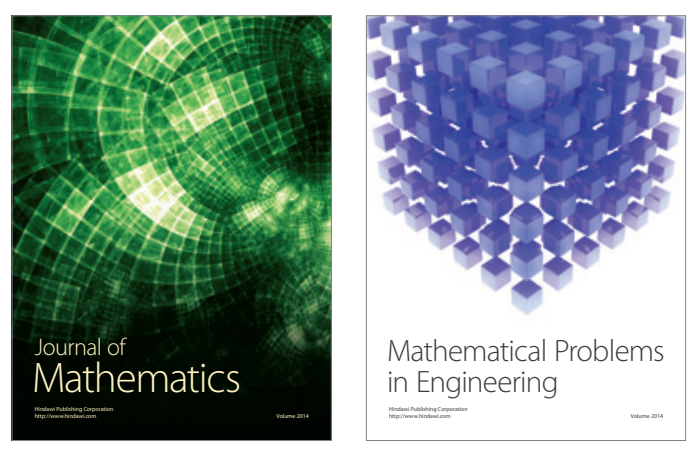

Mathematical Problems in Engineering
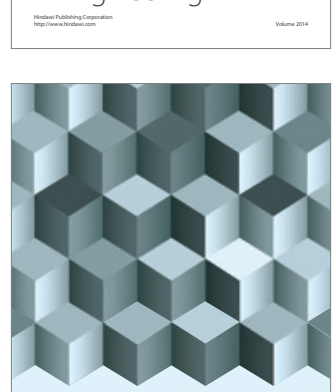

Journal of

Function Spaces
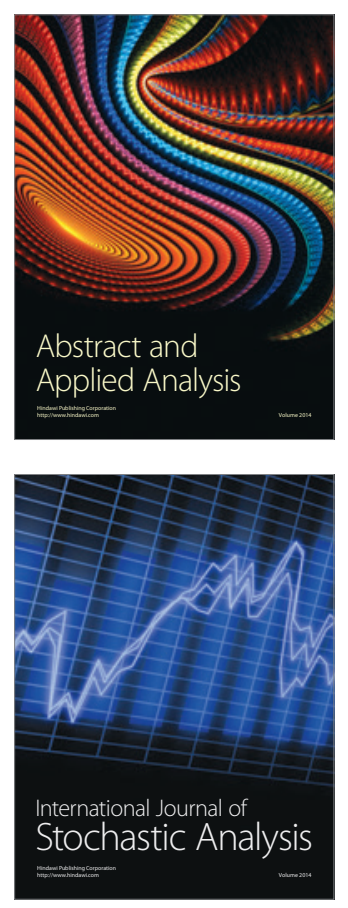

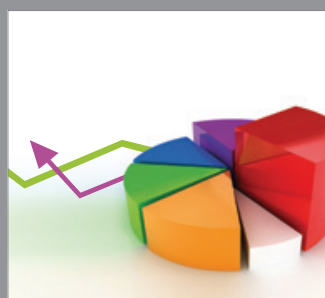

ournal of

Probability and Statistics

Promensencen
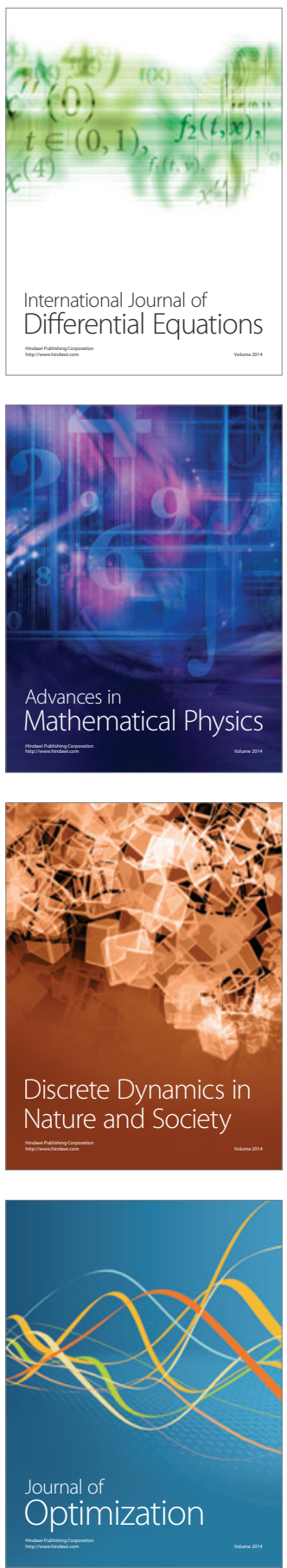